\title{
Hybrid Particle Swarm Optimization Based Optimal Reactive Power Dispatch
}

\author{
P. Subbaraj \\ Principal \\ Theni Kammavar Sangam \\ College of Technology, Theni, \\ Tamilnadu
}

\author{
P. N. Rajnarayanan \\ Asst. Prof / EEE \\ Theni Kammavar Sangam College \\ of Technology, Theni, Tamilnadu
}

\begin{abstract}
In this paper, a two-phase hybrid particle swarm optimization (PSO) approach is used to solve optimal reactive power dispatch (ORPD) problem. In this hybrid approach, PSO is used to explore the optimal region and direct search is used as local optimization technique for finer convergence. The performance of the proposed hybrid approach is demonstrated with the IEEE 30-bus and IEEE 57-bus systems and also the performance of this hybrid PSO is compared with that of PSO, Evolutionary Programming (EP) and hybrid EP. The performance of the proposed method is compared with the previous approaches reported in the literature. The performance of hybrid PSO seems to be better in terms of solution quality and computational time.
\end{abstract}

\section{Keywords}

Particle swarm optimization, evolutionary programming, direct search, optimal reactive power dispatch, hybrid approach.

\section{INTRODUCTION}

The Optimal Reactive Power Dispatch problem is a non-linear optimization problem with many uncertainties. The loads acquire reactive power for magnetizing purposes at no load conditions. The electric power loads vary from hour to hour. The change of load causes variation in the reactive power requirement. The reactive power will depend on voltage, so that the variation of load causes the variation of voltage. Hence the important operating task is to maintain the voltage within the allowable range for high quality consumer service. The objective of the ORPD is to minimize the system real power loss. This objective can be achieved by employing the various reactive compensation devices such as automatic voltage regulators (AVRs), tap changing transformers and shunt capacitors/reactors [1].

A wide variety of conventional optimization techniques such as linear programming, Newton approach, interior point methods and dynamic programming [2-6] have been developed to solve ORPD problem. Generally these techniques suffer due to algorithmic complexity, insecure convergence, and sensitivity to initial search point. [7].

The expert systems [8], fuzzy logic [9], AI approach [10], fuzzy linear programming [11], evolutionary programming (EP)
[12], are some of the heuristic techniques that have been used, recently, to solve the ORPD problem. The EP is suitable for solving global optimization problems like ORPD. The only disadvantage of EP is that it takes more computation time [13].

This paper proposes a hybrid approach to the optimal reactive power dispatch problem. Particle Swarm Optimization (PSO) is one of the evolutionary computation (EC) technique based on swarm intelligence. It is sensitive to the tuning of its parameters and has a flexible mechanism to explore a global optimum point within a short calculation time [14].

By employing the PSO initially the solution quality improves rapidly; later on obtaining the further improvement is very difficult and most of the computation time is spend over obtaining small improvements. To overcome this problem PSO is used for initial exploration and the local search (LS) technique is employed for finer convergence. The convergence of LS techniques depends on the initial search point and quickly finds the local optimum if the starting point is nearer to the optimum [15]. This paper employs direct search (DS) [16] as a LS technique.

The hybrid approach consists of two phases. In phase-1, PSO is employed to obtain the optimal region quickly and in phase-2, the DS with systematic reduction of the size of the search region [16] is used to find the local optimum. To validate the proposed hybrid method, it is tested on two IEEE standard test systems having non-linear characteristics. The results of the proposed hybrid approach are compared with PSO, EP and hybrid EP. The comparison exhibits the effectiveness of the proposed approach in terms of solution quality and computation time.

This paper is organized as follows: The reactive power dispatch problem is introduced in section 2. The swarm technique is briefed in section 3. The direct search technique is introduced in section 4. The hybrid approach is explained in section 5. Test cases and numerical solutions are presented in section 6 . Conclusions are drawn in section 7.

\section{PROBLEM FORMATION}

The purpose of the ORPD is to minimize the system real power losses. The general ORPD with normal power system condition can be formulated [9] as follows:

The objective function is represented as: 


$$
\operatorname{Min} P_{L}=\sum_{k=1}^{n l} \operatorname{Loss} k
$$

where,

$$
\begin{array}{ll}
\mathrm{P}_{\mathrm{L}} & =\text { network real power loss } \\
\mathrm{nl} & =\text { number of lines }
\end{array}
$$

The power loss is a non-linear function of bus voltages, which are functions of control variables. The minimization problem is subject to operating constraints [9], which are limits on various control variables (the inequality constraints) and power flow constraints (the equality constraints).

Equality constraints:

$$
\begin{aligned}
& 0=P_{i}-V_{i} \sum_{j \in N_{i}} V_{j}\left(G_{i j} \cos \theta_{j j}+B_{j j} \sin \theta_{j j}\right) i \varepsilon N B-1 \\
& 0=Q_{i}-V_{i} \sum_{j \varepsilon N_{i}} V_{j}\left(G_{i j} \sin \theta_{i j}-B_{i j} \cos \theta_{j j}\right) \quad i \varepsilon N P Q
\end{aligned}
$$

where,

$$
\begin{aligned}
V_{i}= & \text { voltage magnitude at } i^{\text {th }} \text { bus } \\
\mathrm{G}_{\mathrm{ij}}, \mathrm{B}_{\mathrm{ij}}= & \text { mutual conductance and susceptance between } \\
& \text { bus } \mathrm{i} \text { and } \mathrm{j} \\
\theta_{\mathrm{ij}} \quad= & \text { voltage angle difference between bus } \mathrm{i} \text { and } \mathrm{j} \\
\mathrm{N}_{\mathrm{B}-1}= & \text { total number of buses excluding slack bus } \\
\mathrm{N}_{\mathrm{PQ}}= & \text { set of PQ buses } \\
\mathrm{N}_{\mathrm{i}} \quad & \text { number of buses }
\end{aligned}
$$

Inequality Constraints:

In the control variables, the generator bus voltages (AVR operating values) are taken as continuous variable; the transformer tap settings are taken as discrete variable and shunt susceptance values are taken as binary variable. The load bus voltages and reactive power generation $\mathrm{Q}_{\mathrm{g}}$ are taken as state variables.

Continuous control variable:

$V_{G}^{\min } \leq V_{G} \leq V_{G}^{\max } ; i \varepsilon N_{G}$

Discrete Control variable:

$T_{k}^{\min _{\leq}} T_{k} \leq T_{k}^{\max } ; \quad k \varepsilon N_{T}$

$B_{s h_{i}}^{\min } \leq B_{s h_{i}} \leq B_{s h_{i}}^{\max } ; \quad i \varepsilon N_{s h}$

State Variables:

$$
\begin{aligned}
& v_{P Q_{i}}^{\min } \leq v_{F Q_{i}} \leq \nu_{F Q_{i}}^{\max } ; \quad i s N_{F Q} \\
& \theta_{G_{i}}^{\min } \leq Q_{G_{i}} \leq Q_{G}^{\operatorname{mx}} ;
\end{aligned}
$$

State variables are restricted by adding them as a quadratic penalty terms to the objective function. Therefore, the equation (1) is changed to the following form: $\min$

$$
F=P_{L}+\sum_{i \Sigma N_{y} \lim }^{\sum} \lambda_{V_{i}}\left(V_{i}-V_{i}^{\lim }\right)^{2}+\sum_{i \Sigma N_{Q} \lim } \lambda_{Q G_{i}}\left(Q_{G_{i}}-Q_{G_{i}}^{\lim }\right)^{2}
$$

where,

$$
\lambda_{V_{i} \&}{ }^{\lambda} Q_{G_{i}} \quad=\text { penalty terms }
$$

$\mathrm{V}_{\mathrm{i}}^{\lim }$ and $Q_{G_{i}}^{\lim }$ in the equation (8) are defined as follows:

$$
\begin{aligned}
& V_{i}^{\lim }=\left\{\begin{array}{ll}
V_{i}^{\min } & \text { if } V_{i}<V_{i}^{\min } \\
V_{i}^{\max } & \text { if } V_{i}>V_{i}^{\max }
\end{array}\right\} \\
& Q_{G_{i}}^{\lim }=\left\{\begin{array}{ll}
Q_{G_{i}}^{\min } & \text { if } Q_{G_{i}}<Q_{G_{i}}^{\min } \\
Q_{G_{i}}^{\max } & \text { if } Q_{G_{i}}>Q_{G_{i}}^{\max }
\end{array}\right\}
\end{aligned}
$$

The objective function of the target power system is calculated using load flow calculation with the above mentioned equality and inequality constraints.

\section{PARTICLE SWARM OPTIMIZATION}

The PSO algorithm was introduced by Kennady and Eberhart [17]. PSO is a swarm intelligence method for global optimization. It is a population based evolutionary algorithm. PSO is basically developed through simulation of bird flocking in two-dimension space. Bird flocking optimizes a certain objective function. It updates the population of individuals according to the fitness information, so that the individuals of population can be expected to move towards better solution areas. The position of each agent is represented by $\mathrm{XY}$ axis position and also the velocity is expressed by vx (the velocity along $\mathrm{X}$ axis) and vy (the velocity along $\mathrm{Y}$ axis). Modification of the agent position is realized by the position and velocity information.

Each agent knows its best value so far (pbest) and its XY position. This information is analogy of personal experiences of each agent. Moreover, each agent knows the best value so far in the group (gbest) among pbests. This information is analogy of knowledge of how other agents around them have performed. Namely, each agent tries to modify its position using the following information:

- the current positions $(\mathrm{x}, \mathrm{y})$; the current velocities (vx, vy); the distance between the current position and pbest; the distance between the current position and gbest.

This modification of positions can be represented by the concept of velocity. 
Velocity of each agent can be modified by the following equation:

$\mathrm{V}_{i}^{\mathrm{K}+1}=w_{\mathrm{i}}^{\mathrm{k}}+\mathrm{c}_{1}$ fand ${ }_{1} \times\left(\mathrm{F}_{\text {best }}-\mathrm{X}_{\mathrm{i}}^{\mathrm{k}}\right)+\mathrm{c}_{2}$ fand ${ }_{2} \times\left(\mathrm{g}\right.$ best $\left.-\mathrm{X}_{i}^{\mathrm{k}}\right)$

where,

$v_{i}^{k}=$ velocity of agent $i$ at iteration $k ; w=$ weighting function; $c_{1}, c_{2}=$ acceleration constants; rand $=$ random number between 0 and $1 ; X_{i}^{k}=$ current position of agent $i$ at iteration $k$; pbest $t_{i}=$ pbest of agent $i$; gbest $=$ gbest of the group.

The following weighting function is usually utilized in (10):

$$
w=w_{\max }-\frac{w_{\max }-w_{\text {min }}}{\text { itet }_{\max }} x \text { itet }
$$

where,

$$
\begin{aligned}
w_{\max } & =\text { initial weight; } w_{\min }=\text { final weight; iter } r_{\max } \\
& =\text { maximum iteration number, }
\end{aligned}
$$

iter $=$ current iteration number.

Using (10) and (11), a certain velocity, which gradually gets close to pbest and gbest, can be calculated. The current position (searching point in the solution space) can be modified by the following equation:

$$
X_{i}^{k+1}=X_{i}^{k}+Y_{i}^{k+1}
$$

\section{DIRECT SEARCH}

The optimization procedure based on direct search is found effective in various problems. This method is very useful for the problems having more than one local optimum. It is used to locate the promising area to get the global minima [16]. This direct search optimization procedure is implemented as follows:

Step1: The best solution vector from the phase-1 of the hybrid approach is used as an initial search point $\mathrm{C}(\mathrm{o})$ for phase-2. The initial range vector is defined as

$\mathrm{R}(\mathrm{o})=$ range multiplication factor $(\mathrm{RMF}) *$ range

where,

range $=$ difference between maximum and minimum values of $\mathrm{C}(\mathrm{o})$

Step2: Generate $\mathrm{N}$ trial solution vectors around $\mathrm{C}(\mathrm{o})$ using:

$\mathrm{C}_{\mathrm{i}}=\mathrm{C}(\mathrm{o})+\mathrm{R}(\mathrm{o}) . *$ rand $(1, \mathrm{n})$

where,

$\mathrm{C}_{\mathrm{i}}=\mathrm{i}^{\text {th }}$ solution vector; $.^{*}=$ element by element multiplication; rand $(1, \mathrm{n}) \quad=$ random vector.

Step3: Calculate the objective function value for each solution vector.

Step4: Find the trial solution set, which minimize the objective $\mathrm{C}(\mathrm{o})=\mathrm{C}_{\text {bes }}$ function and call it as $\mathrm{C}_{\text {best }}$ and equate it to $\mathrm{C}(\mathrm{o})$ :

Step5: Reduce the range by an amount given by:

$\mathrm{C}(\mathrm{o})=\mathrm{C}(\mathrm{o}) *(1-\beta)$

where,

$\beta=$ range reduction factor

Step6: The algorithm proceeds to step2, unless it meets the stopping criterion.

\section{HYBRID ALGORITHM FOR RPC}

In phase-1 of the hybrid algorithm, PSO is employed to explore the whole search space and in phase-2, the DS is applied.

Phase-1 algorithm

Step1: Initialization: Initial searching vectors and velocities are generated randomly.

Step2: The objective function for each particle in the initial population is evaluated using load flow calculation. $p_{\text {best }}$ is set to each initial searching point. The initial bestevaluated value among $p_{\text {bests }}$ is set to $g_{\text {best }}$

Step3: Velocity updating: Using the global best and individual best of each particle, particle velocity is updated according to (10)

Step4: Position Updating: Based on the updated velocities, each particle changes its position according to the (12).

Step5: The objective function to the new searching points and the evaluation values are calculated. If the evaluation value of each agent is better than the previous $p_{\text {best }}$ value, the value is set to $p_{\text {best }}$. If the best $p_{\text {best }}$ is better than $g_{\text {best }}$, the value is set to $g_{\text {best. }}$ All of $g_{\text {bests }}$ are stored as candidates for the final control strategy.

Step6: If the stopping criterion is met, then go to Step 7.

Otherwise, go to Step 3.

Step7: The phase-2 algorithm (given in section 4) is invoked.

\section{TEST RESULTS AND ANALYSIS}

The performance of the evolutionary algorithms PSO and EP and their hybrids called hybrid PSO and hybrid EP are evaluated. In hybrid EP, the phase-1 employs EP and phase-2 employs DS.

The following is the stopping criterion for all the algorithms:

$|\mathrm{P}(\mathrm{i})-\mathrm{P}(\mathrm{i}-1)| /|\mathrm{P}(\mathrm{i})| \leq \varepsilon$,

where,

$\mathrm{P}(\mathrm{i})=$ value at current iteration; $\mathrm{P}(\mathrm{i}-1)=$ value at previous iteration;

$\varepsilon=$ is a sufficiently small positive value and taken as $10^{-5}$.

\subsection{Description of the test systems}

In order to validate the proposed hybrid approach, it is tested with two test systems having non-linear characteristics. The first test system is IEEE 30 bus system. It consists of six generator buses, 24 load buses and 41 branches in which 4 branches are tap changing transformers branches. In addition, buses 10, 12, 15, 17, $20,21,23,24$ and 29 have been selected as shunt VAR compensation buses. In IEEE 30 bus system, totally 19 control variables are taken for reactive power dispatch. The branch parameters and loads were taken from [18]. The total loads are $\mathrm{P}_{\text {load }}=2.834$ [p.u] and $\mathrm{Q}_{\text {load }}=1.262[\mathrm{p} . \mathrm{u}]$. The initial transmission line loss is 0.05817 [p.u].

The second test system is IEEE 57 bus system. It has seven generator buses, 50 load buses and 80 branches in which 17 branches are tap changing transformer branches. In addition, buses 18, 25 and 53 have been selected as shunt VAR 
compensation buses, totally 27 control variables are taken for consideration in IEEE 57 bus system. The branch parameters and loads are taken from [19]. The total loads are $\mathrm{P}_{\text {load }}=12.508$ [p.u] and $\mathrm{Q}_{\text {load }}=3.364$ [p.u]. The initial transmission line loss is 0.2793 [p.u]. In both test systems, the base MVA is taken as 100. Table I gives the different control variables settings for the two test systems.

Table I:

Control Variable Settings for the Two Test Systems

\begin{tabular}{|c|c|c|c|}
\hline Test Cases & Variables & Min [p.u] & Max [p.u] \\
\hline \multirow{4}{*}{30} & $\mathrm{~V}_{\mathrm{G}}$ & 0.95 & 1.10 \\
\cline { 2 - 4 } & $\mathrm{V}_{\mathrm{PQ}}$ & 0.95 & 1.05 \\
\cline { 2 - 4 } & $\mathrm{T}$ & 0.90 & 1.10 \\
\cline { 2 - 4 } & $\mathrm{B}_{\mathrm{sh}}$ & 0.0 & 0.05 \\
\hline \multirow{4}{*}{57} & $\mathrm{~V}_{\mathrm{G}}$ & 0.95 & 1.10 \\
\cline { 2 - 4 } & $\mathrm{V}_{\mathrm{PQ}}$ & 0.95 & 1.05 \\
\cline { 2 - 4 } & $\mathrm{T}$ & 0.90 & 1.10 \\
\cline { 2 - 4 } & $\mathrm{B}_{\mathrm{sh}} 18$ & 0.0 & 0.100 \\
\cline { 2 - 4 } & $\mathrm{B}_{\mathrm{sh} 25}$ & 0.0 & 0.059 \\
\cline { 2 - 4 } & $\mathrm{B}_{\mathrm{sh} 53}$ & 0.0 & 0.063 \\
\hline
\end{tabular}

\subsection{Parameter Selection}

Table II gives the parameters used in different EC techniques and in direct search method.

Table II:

Parameters Used in EC techniques and Direct Search Method

\begin{tabular}{|l|c|c|c|}
\hline $\begin{array}{l}\text { Algorithm / } \\
\text { Particulars }\end{array}$ & PSO & EP & Direct Search \\
\hline Population size & 10 & 10 & - \\
\hline $\mathrm{C}_{1}, \mathrm{C}_{2}$ & 2,2 & - & - \\
\hline $\mathrm{w}_{\max }, \mathrm{w}_{\min }$ & $0.9,0.4$ & - & - \\
\hline Scaling factor & - & 0.005 & - \\
\hline $\mathrm{RMF}$ & - & - & 0.5 \\
\hline$\beta$ & - & - & 0.05 \\
\hline
\end{tabular}

\subsection{Testing Strategies}

Since the proposed approach is the hybridization of PSO and DS, it is necessary to find the relative strength of each constituent. Four testing strategies are carried on the test systems.

1) The EP is applied

2) The classical PSO is applied.

3) The hybrid EP is applied.

4) The proposed hybrid PSO is applied.

The coding is written on MATLAB 6.5 package and executed in Pentium IV, $1.5 \mathrm{GHz}$, and $128 \mathrm{MB}$ RAM processor.

\subsection{Results and Discussion}

The convergence characteristics of all strategies for two test systems are given in Fig.1 and Fig.2.

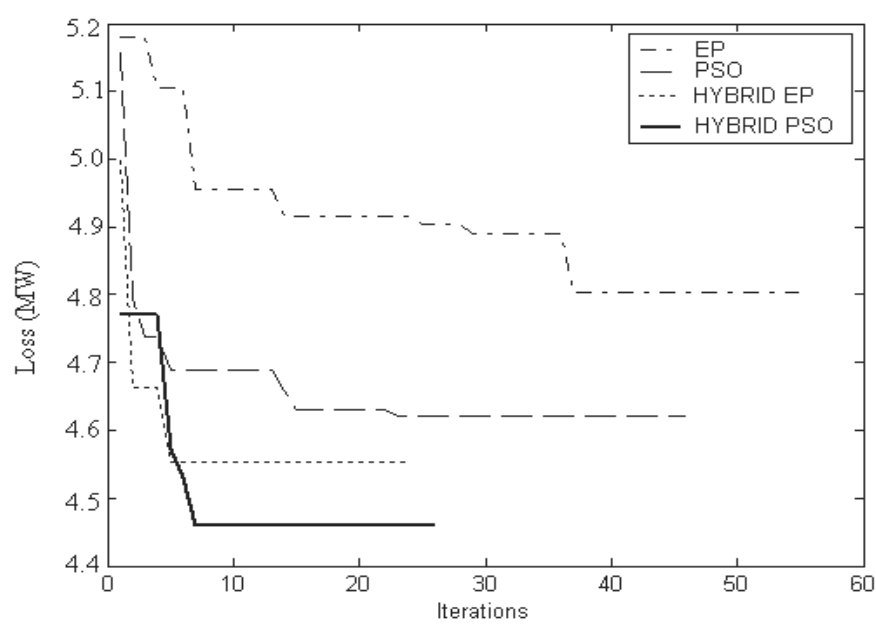

Figure 1. Convergence characteristics for IEEE 30 bus system

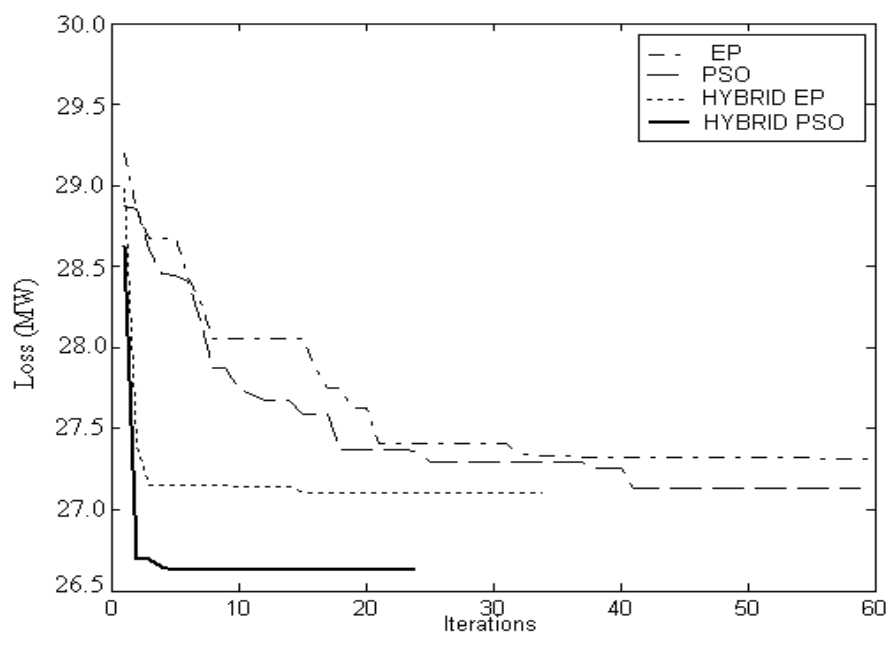

Figure2. Convergence characteristics for IEEE 57 bus system

From the convergence characteristics, it is learnt that PSO performs better than EP in terms of solution quality. For the first test case, EP takes around 35 iterations to converge, whereas the PSO takes only 25 iterations to converge. In the second test, the EP takes 30 iterations to converge; the PSO takes 40 iterations to converge. In both the test cases the PSO obtains the best solution than EP.

The PSO is one of the evolutionary algorithms that do not use the survival of fittest concept. The PSO has no evolutionary operators such as crossover and mutation thereby the PSO is faster than EP. The PSO has the memory i.e., each particle is varied according to its past experience and relationship with other particles in the population which ensures near optimal solutions.

Though the performance of PSO seems to better, almost there is no further improvement in solution quality and time is wasted in computation without improvement for many iterations. In general local search techniques have the advantage of solving the optimization problem quickly, though the results are very much dependent on the initial starting point; therefore they can easily be 
trapped at a local optimum [15]. Hence if one can make use of the advantages of both local search and PSO (in general evolutionary techniques) technique, the optimization algorithm can be made both effective and efficient. When the direct search (local search) technique with systematic reduction in size of the search region [16] is combined with the PSO and EP, the solution quality is improves than the PSO and EP strategies. The convergence characteristics clearly demonstrate the effectiveness of the hybrid approach over the EP and PSO techniques as it reaches the local optimal point with in 10 iterations. For all the two test cases, the hybrid PSO converges faster and rank first in the performance scale.

The Table III gives the loss values in MW and computation time in seconds. In the IEEE 30 bus system EP obtains 17.01\% loss reduction, where as the PSO obtains $20.17 \%$. With hybrid EP the loss reduction is $21.29 \%$ whereas for the hybrid PSO, the loss reduction is $23.76 \%$. The hybrid techniques are able to obtain better solution in lesser computational time. For hybrid PSO the computation time is around $11 \mathrm{sec}$. For PSO, EP and hybrid EP the computational time is $47 \mathrm{sec}, 68 \mathrm{sec}$ and $15 \mathrm{sec}$ respectively. The hybrid PSO is faster than PSO by $36 \mathrm{sec}$, EP by $57 \mathrm{sec}$ and hybrid EP by $5 \mathrm{sec}$.

Table III:

\section{Loss Values and Computational Time}

\begin{tabular}{|c|c|c|c|c|c|}
\hline \multirow{3}{*}{$\begin{array}{c}\text { Loss } \\
\text { values } \\
{[\mathrm{MW}]}\end{array}$} & Method & EP & PSO & $\begin{array}{l}\text { HYBRID } \\
\text { EP }\end{array}$ & $\begin{array}{c}\text { HYBRID } \\
\text { PSO }\end{array}$ \\
\hline & $\begin{array}{c}\text { IEEE } 30 \\
\text { bus }\end{array}$ & 4.8275 & 4.6434 & 4.5782 & 4.4345 \\
\hline & $\begin{array}{c}\text { IEEE } 57 \\
\text { bus }\end{array}$ & 27.4230 & 27.2522 & 27.2132 & 26.5890 \\
\hline \multirow{2}{*}{$\begin{array}{c}\text { Comp } \\
\text { utation } \\
\text { al time } \\
{[\mathrm{sec}]}\end{array}$} & $\begin{array}{c}\text { IEEE } 30 \\
\text { bus }\end{array}$ & 68.73 & 47.12 & 15.74 & 11.45 \\
\hline & $\begin{array}{c}\text { IEEE } 57 \\
\text { bus }\end{array}$ & 112 & 68.56 & 27.52 & 18.38 \\
\hline
\end{tabular}

In the IEEE 57 bus system EP obtains $1.81 \%$ loss reduction, whereas for the classical PSO, the loss reduction is $2.42 \%$. For the hybrid PSO saving is $4.80 \%$. The PSO is faster than EP by $43 \mathrm{sec}$. The hybrid PSO is $94 \mathrm{sec}$ (6 times) faster than EP and $50.17 \mathrm{sec}$ (4 times) than PSO and $9 \mathrm{sec}$ faster than hybrid EP.

From the above facts it can be inferred that the performance of hybrid PSO is better than other methods PSO, EP and hybrid EP in terms of solution and computational time.

\subsection{Robustness test}

The performance of the EC techniques cannot be judged by single run. If the algorithm gives consistent results, then the algorithm said to be robust. The 100 trial runs were performed for both the test systems. The results for the two test systems are given in Tables IV and V.

Table IV:

Comparison of Four Testing Strategies for IEEE 30 bus System

\begin{tabular}{|l|c|c|c|c|}
\hline Loss (MW) & EP & PSO & HYBRID EP & HYBRID \\
\hline
\end{tabular}

\begin{tabular}{|l|c|c|c|c|}
\hline & & & & PSO \\
\hline Best & 4.8275 & 4.6434 & 4.5782 & $\mathbf{4 . 4 3 4 5}$ \\
\hline Worst & 4.9681 & 4.7823 & 4.6682 & $\mathbf{4 . 4 8 0 1}$ \\
\hline Average & 4.8723 & 4.6863 & 4.5987 & $\mathbf{4 . 4 6 3 2}$ \\
\hline
\end{tabular}

Table V:

Comparison of Four Testing Strategies for IEEE 57 bus System

\begin{tabular}{|l|c|c|c|c|}
\hline $\begin{array}{c}\text { Loss } \\
(\mathrm{MW})\end{array}$ & EP & PSO & HYBRID EP & HYBRID PSO \\
\hline Best & 27.4230 & 27.2532 & 27.2132 & $\mathbf{2 6 . 5 8 9 0}$ \\
\hline Worst & 27.6631 & 27.3763 & 27.3581 & $\mathbf{2 6 . 6 3 8 0}$ \\
\hline Average & 27.5230 & 27.2931 & 27.2798 & $\mathbf{2 6 . 6 1 3 0}$ \\
\hline
\end{tabular}

The results show the superiority of the hybrid PSO over the other testing strategies. The average value obtained by the hybrid PSO is always very close to the best value and less than the best values of others strategies. It shows the robustness of the proposed approach.

Tables VI and VII show the consistency of the proposed approach in achieving the minimum objective function for the two test systems. The success rate of the proposed hybrid PSO approach is higher (74\%) than other strategies (58\% for PSO, $63 \%$ for EP and 67 for hybrid PSO) for the first test system.

Table VI:

Frequency of Convergence for IEEE 30 bus System

\begin{tabular}{|l|c|c|c|c|}
\hline \multirow{2}{*}{ Strategies } & \multicolumn{4}{|c|}{ Loss Range (MW) } \\
\cline { 2 - 5 } & $4.40-4.55$ & $4.55-4.65$ & $4.65-4.85$ & $4.85-5.00$ \\
\hline EP & 0 & 0 & 63 & 37 \\
\hline PSO & 0 & 58 & 42 & 0 \\
\hline HYBRID EP & 0 & 67 & 33 & 0 \\
\hline HYBRID PSO & $\mathbf{7 4}$ & $\mathbf{2 6}$ & $\mathbf{0}$ & $\mathbf{0}$ \\
\hline
\end{tabular}

Table VII:

Frequency of Convergence for IEEE 57 bus System

\begin{tabular}{|l|c|c|c|c|c|}
\hline \multirow{3}{*}{ Strategies } & \multicolumn{5}{|c|}{ Loss Range (MW) } \\
\cline { 2 - 6 } & $\begin{array}{c}26.40- \\
26.60\end{array}$ & $\begin{array}{c}26.60- \\
27.00\end{array}$ & $\begin{array}{c}27.00- \\
27.30\end{array}$ & $\begin{array}{c}27.30- \\
27.45\end{array}$ & $\begin{array}{c}27.45- \\
27.85\end{array}$ \\
\hline EP & 0 & 0 & 0 & 56 & 44 \\
\hline PSO & 0 & 0 & 72 & 28 & 0 \\
\hline HYBRID EP & 0 & 0 & 68 & 32 & 0 \\
\hline $\begin{array}{l}\text { HYBRID } \\
\text { PSO }\end{array}$ & $\mathbf{7 7}$ & $\mathbf{2 3}$ & $\mathbf{0}$ & $\mathbf{0}$ & $\mathbf{0}$ \\
\hline
\end{tabular}

The above statement is true for the second test system also. The success rate of proposed hybrid PSO approach is higher (77\%) than other strategies $(72 \%$ for PSO, $56 \%$ for EP and $68 \%$ for hybrid EP) for the IEEE 57 bus system. From the Table 8 and 9, it can be said that the hybrid PSO always minimizes the objective function. Even though the PSO, EP and hybrid EP display similar characteristics, the hybrid approach converges to the optimal value when other methods premature. 


\subsection{Comparison of Hybrid PSO with other techniques}

The proposed hybrid method is compared with Multi Agent based Particle Swarm Optimization (MAPSO) [20] and Interior Point (IP) [21] for IEEE 30 bus system is given in Table VIII.

Table VIII:

Comparisons for IEEE 30 bus system

\begin{tabular}{|l|c|c|c|}
\hline Loss (MW) & HYBRID PSO & MAPSO & IP \\
\hline Best & $\mathbf{4 . 4 3 4 5}$ & 4.8747 & 5.1009 \\
\hline
\end{tabular}

From the Table VIII, the loss value obtained by the proposed method is much lesser than the MAPSO and IP methods.

\section{CONCLUSION}

A two-phase hybrid PSO method is proposed for optimal reactive power dispatch problem. The phase-1 uses the classical PSO, while local optimization by direct search is applied in phase-2. In order to validate the proposed approach, it is tested with two standard test systems having non-linear characteristics and the results are compared with other techniques reported in the literature. The proposed hybrid PSO is obtains lesser loss values than other strategies with lesser computational time. The robustness test is also conducted to verify the consistency of the proposed approach. It is observed from the repeated trial runs, the hybrid PSO approach always converged to near optimal solution. The test results show that, the proposed approach not only improves the solution quality but reduces the computation time also and suitable for optimal reactive power dispatch problem.

\section{REFERENCES}

[1] Bansal. R., Bhatti, T., and Kothari, D. 2003 Artificial intelligence techniques for reactive power/voltage control in power systems: A review International Journal of power and energy systems, 23 (2003), 81-89.

[2] Alsac, O., Bright, J., Prais, M., Scott B. and Marinho, J.L. 1990. Further developments in LP based optimal power flow IEEE Transactions on Power Systems, 5 (1990), 697-711.

[3] Sun, D.I., Ashley, B., Brewar, B., Hughes, A. and Tinny, W.F. 1984 Optimal power flow by Newton approach. IEEE Transactions on Power Apparatus and systems, 103 (1984), 2864-2880.

[4] Granville, S., 1994. Optimal reactive power dispatch through interior point methods IEEE Transactions on Power Systems 9 (1994), 136-146.

[5] Hong, Y.Y. Sun D.I., Lin S.Y. and Lin, C.J. 1990 Multi-year multi-case optimal VAr planning IEEE Transactions on Power Systems, 5 (1990), 1294-1301.

[6] Lu, F.C. and Hsu, Y.Y. 1995 Reactive power/voltage control in a distribution substation using dynamic programming IEE Proceedings on Generation, Transmission and Distribution, 142 (1995) 639-645.
[7] Abido, M.A. 2002 Optimal power flow using particle swarm optimization International journal of Electric Power and Energy Systems, 24 (2002), 563-571.

[8] Cheng, S.J., Malik O.P., and Hope, G.S. 1988 An expert system for voltage and reactive power control of a power system IEEE Transactions on Power Systems, (1988) 14491455

[9] Abdul Rahman, K.H. and Shahidehpour, S.M. 1993 A fuzzybased optimal reactive power control IEEE Transactions on Power Systems, 8 (1993) 662-670.

[10] Abdul Rahman K.H., Shahidehpour S.M. and Daneshdoost M., 1995 AI approach to optimal VAR control with Fuzzy reactive loads IEEE Transactions on Power Systems, 10 (1995) 88-97.

[11] Tomsovic, K. 1992 A fuzzy linear programming approach to the reactive power/voltage control problem IEEE Transactions on Power Systems, 7 (1992) 287-293.

[12] Wu, Q. and Ma, J.T. 1995 Power systems optimal reactive power dispatch using evolutionary programming IEEE Transactions on Power Systems, 10 (1995) 1243-1249.

[13] Ma, J.T. and Lai, L.L. 1996 Evolutionary programming approach to reactive power planning IEE Proceedings on Generation, Transmission and Distribution, 143 (1996) 365370 .

[14] Liang, J.J., Qin, A.K Suganthan P.N. and Baskar S.,2004 Particle swarm optimization algorithms with novel learning strategies IEEE International Conference on. Systems, Man and Cybernetics, (2004) 3659-3664.

[15] Baskar, S., Subbaraj P. and Rao, M.V.C. 2003 Hybrid real coded genetic algorithm solution to economic dispatch problem International Journal of computers and electrical engineering, (2003) 407-419.

[16] Luss, R. and Jaakola, T.H.I. 1973 Optimization by direct search and systematic reduction of the size of the search region American Institute of Chemical Engineers Journal, 19 (1973) $760-766$.

[17] Kennady, J. and Eberhart, R.C. 1995 Particle swarm optimization IEEE International Conference on Neural networks, (1995) 1942-1948.

[18] Lee, K., Park Y. and Ortiz, J. 1985 A united approach to optimal real and reactive power dispatch IEEE Transactions on Power Apparatus and systems, 104 (1985), 1147-1153.

[19] http://www.ee.washington.edu/research/pstca/pf57/pg tca57 bus.htm

[20] Zhao, B. Guo, C.X. and Cao, Y.J. 2005 A multiagent-based particle swarm optimization approach for optimal reactive power dispatch IEEE Transactions on Power Systems, 2 (2005), 1070-1078.

[21] de Souza A. C. Z., Honorio, L. M. Torres, G. L. and Lambert-Torres, G. 2004 Increasing the loadability of power systems through optimal-local control actions IEEE Trans. Power Syst., 19 (2004), no. 1, pp. 188-194. 